\title{
UN ACERCAMIENTO A LA SAGA FAMILIAR DEL SIGLO XX DE ESPAÑA
}

\author{
SHAOFAN REN \\ Universidad de Estudios Internacionales de Zhejiang, China
}

\begin{abstract}
Resumen. La saga familiar es un tipo de novela cuya escena principal se ubica en una familia. Este género literario no es desconocido ni en el campo de la crítica ni entre los lectores, sin embargo, el estudio correspondiente en España es muy escaso. El presente trabajo, centrado en este tema, se dedica a presentar brevemente el desarrollo del género de la saga familiar de España del siglo XX y analizar algunos de sus rasgos más prominentes característicos para este género literario durante los años 40 y 50, cuando España vive un periodo muy complicado en su historia.
\end{abstract}

Palabras clave: literatura española, novela, familia, saga familiar, siglo XX

\section{INTRODUCCIÓN}

De forma general la saga familiar se entiende como un tipo de novela cuya escena principal discurre en una familia con varias generaciones y suele narrar las vicisitudes que ha experimentado o está experimentando en sí misma o con familias relacionadas. Este género no es muy desconocido ni en el ámbito de la crítica ni entre los lectores, ya que tenemos muchas obras representativas con fama mundial, tales como Los Buddenbrook (Mann, 2008); La saga de los Forsyte (Galsworthy, 2014); Las uvas de la ira (Steinbeck, 2012) y Cien años de soledad (Márquez, 2013). En España también hay un gran número de sagas familiares, aunque los estudios correspondientes son muy escasos y este género literario todavía no se ha analizado en su conjunto. En este trabajo se intenta introducimos brevemente la saga familiar del siglo XX de España así como sus características más destacadas.

\section{EL ORIGEN DE LA SAGA FAMILIAR}

La entrada de la familia en la literatura es muy temprana, pudiéndose remontar a la mitología griega, la Biblia y la mitología nórdica. El mundo de los dioses griegos es un mundo protagonizado por una amplia familia que comienza con Urano y Gea; la migración de Abraham con su esposa y los descendientes que se relata en el Antiguo Testamento también se puede entender como una historia 
de familia. Vale la pena mencionar que el tema del fratricidio en la Biblia se adapta y se refleja en varias novelas posteriores, como Los Abel (Matute, 2014), en la que a través de la historia de Caín y Abel, la autora alude al rencor fratricida entre los republicanos y los nacionales durante la guerra civil, prolongado hasta la posguerra. Si lo vemos desde este punto de vista, ambos nacimientos literarios occidentales, tanto la mitología griega como la Biblia, tienen algo que ver con la saga familiar de la que ahora hablamos.

Además de la mitología griega, en otro sistema mitológico que nace unos siglos más tarde, la mitología nórdica, también hay historias familiares y es exactamente de aquí de donde deriva el nombre de este género: Saga familiar. La difusión original de la mitología nórdica era de forma oral y fue escrita durante los siglos XIII y XIV, principalmente en Edda y Saga. Fue escrita sobre todo en Edda, desde donde tenemos la mayoría de los conocimientos sobre la cosmología y el sistema de los dioses en la mitología nórdica. La Saga tiene mucho que ver con la hagiografía y la historiografía, que registra los relatos sobre los héroes y los reyes según los diferentes géneros, y entre todos los géneros de Sagas de obispos; Sagas de los reyes; Sagas arcaicas y Sagas caballerescas... Destacan las Sagas islandesas (Shao, 1993: 5), que:

relatan las vidas y enfrentamientos de personajes y familias enteras durante la denominada Edad de las Sagas, entre 930 y 1030. Dentro de éstas, las hay de carácter histórico, mientras que otras unen realidad histórica y ficción; otras en las que predomina lo ficticio e incluso algunas que son obras de ficción sin base histórica comprobable. (Astvaldsson, 2015)

Entre las Sagas islandesas, la Saga de familia sobresale no solo por su gran número sino también por su excelente cualidad. Sus mayores representantes son la Saga de Njál; la Saga de Laxdœla, que narra la venganza duradera y repetidora entre varias generaciones de algunas familias y la Saga Egjls, que cuenta las aventuras y la vida cotidiana de una familia con cuatro generaciones (Bernárdez, 1984: 167). En la sociedad primitiva de los clanes, las pequeñas disputas entre personas solían convertirse en conflictos en defensa del honor familiar y si había muertes o heridas, la venganza se difundía como un acto heroico. Por otra parte, los primeros habitantes de Islandia, los colonizadores noruegos de noble linaje, sentían mucha nostalgia y seguían estando orgullosos de sus hazañas familiares, por lo tanto relataban esas historias en repetidas ocasiones. Estas historias familiares tal vez sean el prototipo de las sagas de familia, ya que muchas de ellas se despliegan en torno al desarrollo o las proezas guerreras de las familias.

Como la mayoría de las sagas que cuentan con más importancia y elogio son sagas de familia, la palabra saga va acercándose al significado único de Saga familiar. En 1922, el escritor británico John Galsworthy pone el nombre The Forsyte Saga a su trilogía que narra la historia de una familia de clase media-alta. Esta es la primera vez que la palabra saga se usa con el significado de saga familiar tal y como lo conocemos en la actualidad. Poco a poco, este uso se acepta hasta 
que a día de hoy la RAE cuenta con una definición específica que la conecta con este género literario, que es 'Relato novelesco que abarca las vicisitudes de varias generaciones de una familia' (En línea 1).

\section{SAGA FAMILIAR DEL SIGLO XX DE ESPAÑA}

En lo concerniente a la saga familiar del siglo XX de España, podemos enumerar bastantes obras pertenecientes a este género literario. Durante la preguerra, tenemos una saga familiar escrita por un autor prestigioso de la generación del 98: La casa de Aizgorri (1900) de Pío Baroja, una novela adaptada desde el teatro y, por consiguiente, compuesta casi por completo por diálogos. Narra la preocupación de una pequeña familia burguesa por las dificultades que está experimentando la fábrica familiar. A través de la figura del hijo, el autor nos transmite su preocupación por la abulia que se veía frecuentemente en la sociedad de entonces. En los años 40, la novela más representativa de la tendencia existencial, $\mathrm{Nada}$ (de Laforet publicada en 1944) es una saga familiar. La autora cuenta la historia de Andrea y coloca la escena principal en la casa de su abuela, donde además de la anciana conviven la tía, el tío, la mujer del tío y la criada. Entre las novelas realistas de ese tiempo, también tenemos una saga familiar muy importante: La ceniza fue árbol (de Ignacio Agustí, publicada durante 1943-1965), sobre todo los primeros dos de los cinco volúmenes que compone esta novela-río: Mariona Rebull y El viudo de Rius, que se publican respectivamente en 1943 y 1944, cuya historia se despliega en torno a las vicisitudes que ha experimentado la familia Ruis y lo que pasa entre el matrimonio recién casado. En los años 50, tenemos Los gozos y las sombras (de Gonzalo Torrente Ballester, publicada durante 1957-1962), Los cipreses creen en dios (de José María Gironella, publicada en 1953) y Mi idolatrado hijo Sisí (de Miguel Delibes, publicada en 1953). Aparte de las mencionadas, muchas sagas familiares de este periodo nacen bajo el lápiz de autoras y muchas de ellas son de la primera generación de autoras de posguerra, tales como Los Abel (de Ana María Matute, publicada en 1948); Nosotros los Rivero (de Dolores Medio, publicada en 1953); La Madama (de Concha Alós, publicada en 1969); La sangre (de Elena Quiroga, publicada en 1952) y Las ataduras (de Carmen Martín Caite, publicada en 1983).

La saga familiar del periodo 1960-1975 que cuenta con más fama y elogios es Ágata, ojo de gato de Caballero Bonald, publicada en 1974. En vez de mirar hacia los problemas sociales, el autor se preocupa más por los problemas éticos. Guarnición de silla (de Alfonso Grosso, publicada en 1970) y La otra casa de Mazón (de Benet Goita, publicada en 1973) también son dos sagas familiares, ambas cuentan la decadencia de grandes familias. Durante la Transición, varias sagas familiares muy apreciables son escritas por autoras, entre ellas algunas pertenecen a la primera generación de autoras de posguerra, como Ana María Matute, quien crea otra saga familiar en los años 70: Fragmentos de interior (1976). La escritora catalana Mercè Rodoreda en su saga familiar Espejo roto (1974) narra la historia de tres generaciones de una familia de Barcelona, a través de la cual 
dirige de nuevo la mirada a la época de la preguerra. La autora de la segunda generación de la posguerra, Marina Mayoral, nos presenta tres novelas familiares a finales de los años 70 y a principios de los 80: Cándida, otra vez (1979); Al otro lado (1980) y La única libertad (1982). Las familias en sus novelas no son las nucleares, sino que son familias más amplias, o mejor dicho, son varios linajes de una familia.

Una vez llegada la democracia, la literatura comienza a presentar un panorama más variado. El primer volumen de la trilogía Verdes valles, colinas rojas de Ramiro Pinilla, La tierra convulsa (1986), parece una historia épica del País Vasco porque narra la historia de dos familias en un marco temporal muy amplio, de más de medio siglo, entre el siglo XIX y el XX. Miguel Delibes crea otra saga familiar, Madera de héroe (1987) en los años 80, fijando la mirada en el mundo de un niño. José Manuel Caballero Bonald también escribe otra saga familiar en los años 80, En la casa del padre (1988). A través del ascenso económico y la decadencia moral de una familia burguesa, el autor se fija otra vez en los problemas éticos. Durante los años 90, No solo el fuego (de Benjamín Prado, publicada en 1999) nos muestra un recorrido de la historia más reciente de España. A través de lo que les ocurre a las cinco generaciones de una familia, cada una encontrada en un contexto peculiar, podemos ver cómo son las personas situadas en diferentes épocas. A finales de siglo, las autoras no se alejan tampoco del campo de la saga familiar. Malena es un nombre de Tango (de Almudena Grandes, publicada en 1994) se concentra en describir tres generaciones de mujeres en una familia.

Aunque hay muchas sagas familiares, el estudio correspondiente sobre este tema es muy reducido. En cuanto a los libros monográficos, después de una búsqueda y consulta minuciosas, tenemos que aceptar que hasta ahora todavía no hay ninguna monografía que estudie la saga familiar de España. En lo concerniente a los estudios académicos, tampoco hay mucho escrito. Cuando ponemos saga familiar en el buscador de Dialnet, solo salen 186 resultados. Al marcar humanidades se reducen a 42 resultados y aun así la mayoría de ellos no tiene nada que ver con la literatura, sino que está muy estrechamente vinculado con las familias reales en la historia. Cuando intentamos la búsqueda otra vez introduciendo la palabra clave novela familiar, nos salen muchos resultados relacionados con la psicología porque este término también es un fenómeno psicológico. Después de reducir la búsqueda al ámbito de las humanidades, solo quedan 125 resultados. Por tanto, este género por un lado tiene estudios correspondientes muy escasos, y por otro lado todavía no se ha analizado como un conjunto.

\section{LAS CARACTERÍSTICAS MÁS DESTACADAS DE LA SAGA FAMILIAR DEL SIGLO XX DE ESPAÑA}

Después de una introducción breve de las sagas familiares españolas del siglo XX, intentamos resumir sus características más destacadas. La primera es que los autores, sobre todo los de los años 40 y 50, prefieren metaforizar la situación del 
país o los problemas sociales a través la historia familiar, en otras palabras, aunque la historia sucede en las familias, lo que verdaderamente quieren transmitirnos es la situación general de toda la sociedad. Tal y como lo que indica el profesor Julio Checa, que afirma que:

la presencia del conflicto familiar no ha permitido únicamente ofrecer un caudal inagotable de tramas, sino que también propondría lecturas simbólicas en claves antropológicas, económicas, políticas o culturales de los individuos y sus modos de organizar la existencia. En este sentido, la representación de la familia como microcosmos social sería, sin lugar a duda, uno de los lanzamientos más recurrentes. (Checa, 2015: 37)

Por ejemplo, En Nada y Los Abel, lo que se ve no son solo dos familias, sino una España oscura y cubierta de heridas y magulladuras durante la inmediata posguerra. Torrente Ballester, en su trilogía Los gozos y las sombras, a través de lo ocurrido a varios linajes, sobre todo a su última generación en un pueblo gallego, de los que destacan la familia del viejo señor de la villa y la familia del nuevo capitalista, el autor nos pinta un panorama de la sociedad de preguerra en la que la fuerza vieja cede inevitablemente al capitalismo. Además de narrar las vicisitudes que ha experimentado la familia Rius y lo que pasa entre el matrimonio recién casado, La ceniza fue árbol de Ignacio Augustí nos presenta también el panorama social, sobre todo el de la burguesía entre finales del siglo XIX y comienzos del $\mathrm{XX}$ en Barcelona. En El viudo de Rius del mismo autor se mencionan también varios acontecimientos históricos, así como los movimientos proletarios que tuvieron lugar en Barcelona durante ese periodo peculiar. De la misma manera, en Los cipreses creen en dios, a través de una familia de clase media y las personas que la rodean, que tienen ideologías variadas, el autor nos presenta un panorama global de la Cataluña en preguerra en lo referente tanto a la vida cotidiana de los ciudadanos como a los acontecimientos históricos y políticos.

Se nota que muchas de estas novelas son de los años 40 y 50, es decir, después de la Guerra Civil, y que el tema muchas veces también va en torno a este acontecimiento. La guerra, junto con sus consecuencias, es como una cicatriz horrible que dolía a los autores y había dejado una influencia enorme en el ámbito de la literatura española. Durante ese tiempo, viendo la miseria que había en toda España y la vida oscura de las personas, 'nuestros autores se unieron para denunciar la situación del país, con la esperanza de que sus obras ayudasen a las clases más bajas' (Langa, 2001: 18). Encontrados en este contexto literario, lo que querían escribir los autores, sea saga familiar o no, muchas veces era la situación del país. Por otra parte, la historia de la familia en algún sentido facilita la narración de la alegoría del país. En primer lugar, lo que cuenta la saga familiar son las vicisitudes de una familia de más de una generación, y a través de la experiencia de estas generaciones, podemos ver verticalmente la historia y las transformaciones de toda la sociedad. En segundo lugar, la miseria, la pobreza, el hambre y el dolor, todos estos sufrimientos se ven más claramente en un espacio 
privado como la familia; en este sentido, colocar la escena en una familia facilita la descripción más real de la sociedad de aquel entonces.

El considerable número de autoras es otra característica de la saga familiar. Aunque los escritores varones todavía toman las riendas en el panorama literario durante el siglo XX, la saga familiar es un género literario muy cultivado por las autoras. Varios estudiosos han indicado que la familia es un tema recurrente en la literatura escrita por mujeres:

La profesora y novelista Carmen Riera destaca por la recurrencia de unos mismos temas: la indagación sobre sí misma, el auto-análisis, el retorno a la infancia, la importancia de los universos cerrados y familiares [...] Otros estudios añaden a estas características la minuciosidad en la descripción de las sensaciones, las referencias a lo doméstico, una mayor riqueza léxica en la adjetivación de los colores. (Ruiz, 1997: 170)

Una de las premisas de este fenómeno es que la posición de las autoras subió a un nivel más alto que nunca durante el siglo XX:

Las transformaciones sociales, ideológicas y políticas, el desarrollo de las ideas feministas, la influencia de otros países, son todos ellos factores que contribuyen a que, por fin, en el siglo XX, las mujeres españolas estén plenamente integradas en la literatura, como escritoras y como lectoras. A lo largo del siglo se va produciendo un proceso gradual de incorporación de la mujer a la escritura, frenado lamentablemente por la guerra civil, que va adquiriendo fuerza en los años de la postguerra hasta legar al "boom" de la literatura femenina de la década de los setenta y ochenta. (ibid.: 170)

Aunque cuenten con una posición relativamente elevada, 'la narrativa y la poesía continúan siendo los más cultivados por las mujeres' (ibid.: 153). La abundancia de autoras y su dedicación a la novela es la premisa que sigue al fenómeno de que muchas sagas familiares se escriban por autoras.

Por otra parte, entre la mujer y la familia hay un vínculo establecido desde hace mucho tiempo que no empieza a debilitarse hasta el siglo XX. Las niñas son educadas para cocinar, lavar, planchar, coser, bordar $y$, sobre todo, para ser unas esposas cariñosas y unas madres abnegadas. Además, tienen una escasa participación en las actividades sociales, políticas y educativas, y sus vidas se limitan a la familia. Aunque la mayoría de estas autoras vienen de la burguesía y tienen un nivel de estudios más o menos alto (incluso universitario), las figuras femeninas bajo sus bolígrafos todavía están muy cerca de la familia.

En segundo lugar, una de las características de la creación literaria femenina consiste en la descripción minuciosa de los sentimientos sensibles, íntimos y de dedicación. El lugar donde se favorece la expresión de estas emociones, en vez de tratarse de los espacios públicos, donde estamos rodeados de desconocidos, compañeros y también colegas con quienes tratamos solo de vez en cuando, es la 
casa donde tenemos a las personas más cercanas de manera más íntima, y delante de ellos podemos expresar nuestro 'yo' verdadero. Por lo tanto, el contexto familiar puede ayudar a las autoras a describir mejor los sentimientos sutiles. Por último, uno de los temas favoritos de las escritoras es el auto-análisis, para el que necesitan mirar hacia atrás con el fin de ver su proceso de crecimiento y cómo el ambiente familiar les ha dejado una influencia en la formación de su carácter.

El tercer carácter más destacado de la saga familiar española consiste en que el modo en orden - desorden - ruina es el preferido por los autores, y casi todas las familias en estas historias se caen al final. Hay dos modelos principales de narración en la saga familiar con respecto a la suerte de las familias: uno es en orden - desorden - ruina, otro es nada - en orden - prosperidad. El primer modelo es el más utilizado, y también el que ha conseguido mayor éxito y más fama en España. Hay que añadir que, en ocasiones, como en la saga familiar española En la casa del padre (Caballero, 1988), estos dos modelos se integran y se cuenta primero el ascenso de la familia y luego su decadencia. Otras veces, aunque se adopta el modelo de decadencia, el periodo de en orden se narra solo a través de los recuerdos y la historia empieza directamente con la familia en sin orden. Como el modelo en orden - desorden - ruina es el más aplicado en las sagas familiares de estos dos países, aquí solo desarrollaremos este modelo.

Primero, el estado de en orden. Este periodo se expresa a través de la prosperidad de la familia, cuya razón de ser consiste mayormente en una vida cómoda y la convivencia armoniosa entre los familiares. La función de las descripciones de este periodo es introducir la personalidad de los protagonistas, explicar la historia de la familia, revelar el contexto social y, lo más importante, contrastarla con una familia en decadencia. Después, el periodo de desorden, el tronco de la narración, con contenidos más abundantes y nutridos. Lo más importante de esta parte son las contradicciones a las que se debe la quiebra del orden. En cuanto al contenido de estas contradicciones, son muy variadas: las económicas, las políticas, las culturales, las acontecidas entre los familiares; las que guardan relación con la familia y el mundo exterior; las ocurridas entre la fuerza nueva y la vieja, las de dentro de una persona, etc. A través de estas contradicciones, se puede ver las transformaciones sociales, los conflictos dentro de la familia, los comportamientos y pensamientos de las personas que se encuentran en algún contexto o periodo peculiar y, sobre todo, la personalidad de los protagonistas. En suma, esta parte es la más importante de la narración, no solo por su extensión sino también por todos los mensajes que nos transmite. Por último, encontramos la ruina, el final de la obra. Después de ver el desorden, este final ya no resulta sorprendente, sino que es muy evidente e imaginable. Aquí se informa del desenlace de los personajes y de la familia, que en muchos casos es la descomposición. $\mathrm{O}$ también puede tratarse de la decadencia de una familia, el fin de una época, la extinción de un fenómeno, etc. 


\section{CONCLUSIONES}

Concluyendo se puede destacar que aunque el estudio correspondiente es muy reducido y este género todavía no se ha analizado como conjunto en España, solo concentrado en el siglo XX, sí que existe un gran número de sagas familiares y muchas de ellas son obras excelentes. Tras analizar una amplia gama de obras de este género, han sido determinadas tres características más destacadas: la primera es metaforizar lo que ocurre en el país a través de la historia de la familia; la segunda es el número considerable de autoras; y el último es la adaptación del modo de decadencia. La familia, además de ser una existencia especial para el ser humano, trata también de un microcosmo social. En este sentido, el estudio del reflejo literario de esta existencia es significativo tanto literaria como culturalmente. Para terminar, se espera que este trabajo pueda inspirar el mayor interés sobre este género literario.

\section{RREFERENCIAS BIBLIOGRÁFICAS}

Astvaldsson, A. (2015) Narraciones históricas que relatan vidas y enfrentamientos de personajes y familias. La jornada. Disponible en https://www.jornada.com.mx/2015/04/19/semanxela.html [Consultado el 16 de mayo de 2017].

Bernárdez, E. (1984) Sagas Islandesas. Madrid: S.L.U. ESPASA.

Checa, J. (2015) Del "yo" al "nosotros": visiones de la familia en la dramaturgia española actual. Anales de la literatura española contemporánea, ALEC, 40 (2): 35-59.

Galsworthy, J. (2014) La saga de los Forsyte. Madrid: REINO DE CORDELIA.

Javier, G. (2002) Contrasentidos: Acercamiento a la novela española contemporánea. Zaragoza: Universidad de Zaragoza.

Langa, M. (2000) Del franquismo a la posmodernidad: la novela española (1975-99) análisis y diccionario de autores. Madrid: Universidad de Alicante.

Ruiz, C. (1997) Panorama de escritoras Españolas II. Cádiz: Edita Servicio de Publicaciones de la Universidad de Cádiz.

Shao, X.D. (1993) From "Saga" to "The Family" - the Origin and Cause of Western Family Novels. Journal of Henan Normal University, 6 (1): 85-89.

\section{RECURSOS DIGITALES}

[En linea1] Disponible en http://www.rae.es. [Consultado el 16 de mayo de 2019].

\section{RECURSOS DE ANÁLISIS EMPÍRICO}

Agustí, I. (1975) Mariona Rebull-El viudo Ruis. Barcelona: Planeta.

Alós, C. (1981) La Madama. Barcelona: Círculo de Lectores, S.A.

Baroja, P. (2000) La casa de Aizgorri. Barcelona: Espasa Libros.

Caballero, J. M. (2007) Ágata, ojo de gato. Barcelona: Seix barral.

Caballero, J. M. (1988) En la casa del padre. Barcelona: PLAZA \& JANÉS.

Delibes, M. (2010) Madera de Héroe. Barcelona: Destino.

Delibes, M. (2019) Mi idolatrado hijo Sisí. Madrid: Austral.

García, G. (2013) Cien años de soledad. Barcelona: Debolsillo.

Gironella, J. M. (2012) Los cipreses creen en Dios. Barcelona: Planeta. 
Grandes, A. (2008) Malena es un nombre de tango. Barcelona: Tusquets.

Laforet, C. (2001) Nada. Barcelona: Destino.

Mann, T. (2008) Los Buddenbrook. Barcelona: Edhasa.

Martín, C. (2010) Fragmento de interior. Madrid: Siruela.

Martín, C. (1983) Las ataduras. Barcelona: Bruguera.

Matute, A. M. (2014) Los Abel. Barcelona: Destino.

Mayoral, M. (2002) La única libertad. Madrid: Alfaguara.

Mayoral, M. (1992) Cándida, otra vez. Madrid: Castalia.

Mayoral, M. (2001) Al otro lado. Madrid: Punto de lectura.

Medio, D. (1997) Nosotros, los Rivero. Barcelona: Destino.

Pinilla, R. (2009) La tierra convulsa. Barcelona: Tusquets.

Prado, B. (1999) No solo el fuego. Madrid: Alfaguara.

Quiroga, E. (1952) La sangre. Barcelona: Destino.

Rodoreda, M. (1989) Espejo roto. Barcelona: Seix Barral.

Steinbeck, J. (2012) Las uvas de la ira. Madrid: Alianza.

Torrente, G. (2001) Los gozos y las sombras. I. El señor llega. Madrid: El Mundo.

Torrente, G. (2001) Los gozos y las sombras. II. Donde da la vuelta el aire. Madrid: El Mundo.

Torrente, G. (2001) Los gozos y las sombras. III. La Pascual triste. Madrid: El Mundo.

\title{
APPROACH TO THE FAMILY SAGA OF THE $20^{\text {TH }}$ CENTURY IN SPAIN
}

\begin{abstract}
Family saga is a type of novel that depicts life of a family. This literary genre is not unknown either in the field of criticism or among readers, however, it has been scarcely studied in Spain. The present article, focused on this topic, intends to provide a brief description of the development of the genre of the family saga in Spain during the 20th century and to analyse some of its most prominent features which were characteristic of this literary genre during the 1940s and 1950s, when Spain experienced a very complicated period in its history.
\end{abstract}

Keywords: Spanish literature, novel, family, family saga, $20^{\text {th }}$ century

ShaoFan Ren (Dra. en Humanidades) Trabaja actualmente en la Universidad de Estudios Internacionales de Zhejiang. Sus intereses académicos incluyen la literatura contemporánea de España y la literatura comparada entre España y China. Correo electrónico: 136339110@qq.com 